\title{
Sickness absence as a measure of health status and functioning: from the UK Whitehall II study
}

\author{
Michael Marmot, Amanda Feeney, Martin Shipley, Fiona North, S L Syme
}

Department of Epidemiology and Public Health, University College London Medical School,

66-72 Gower Street, London WC1E 6EA

M Marmot

A Feeney

M Shipley

Department of Preventive and Social Medicine,

University of Otago,

Dunedin,

New Zealand

F North

School of Public

Health,

University of

California,

Berkeley

CA 94720, USA

$S$ L Syme

Correspondence to: Professor M Marmot.

Accepted for publication September 1995

\begin{abstract}
Study objective-To investigate the relationship between self reported health status and sickness absence.

Design-Analysis of questionnaire and sickness absence data from the first phase of the Whitehall II study - a longitudinal study set up to investigate the degree and causes of the social gradient in morbidity and mortality.
\end{abstract}

Setting - London offices of 20 civil service departments.

Participants-Altogether 6895 male and 3413 female civil servants aged 35-55 years. Analysis was conducted on $88 \%$ of participants who had complete data for the present analysis.

Main results - A strong inverse relation between the grade of employment (measure of socioeconomic status) and sickness absence was observed. Men in the lowest grade had rates of sickness absence six times higher than those in the highest grade. For women the corresponding differences were two to five times higher, In general, the longer the duration of absence, the more strongly did baseline health predict rates of absence. However, the health measures also predicted shorter spells, although to a lesser extent. Job satisfaction was strongly related to sickness absence with higher rates in those who reported low job satisfaction. After adjusting for health status the association remained for one to two day absences, but was greatly reduced for absences longer than three days.

Conclusion-There was a strong association between ill health and sickness absence, particularly for longer spells. The magnitude of the association may have been underestimated because of the strength of the association between grade of employment and sickness absence. It is proposed that sickness absence be used as an integrated measure of physical, psychological, and social functioning in studies of working populations.

( $\mathcal{F}$ Epidemiol Community Health 1995;49:124-130)

The costs of sickness absence to government and industry are substantial. In the United Kingdom, more than 370 million working days are lost each year due to certified incapacity, ${ }^{1}$ with a cost to British business estimated at $£ 13$ billion. $^{2}$

Sickness absence is not always thought to be a reliable indicator of morbidity. ${ }^{3}$ An impolite view has been that non-attendance for work has more to do with absence than with sickness and can best be explained by concepts such as the absence culture, ${ }^{45}$ where sickness absence is viewed as a voluntary behaviour, influenced by factors such as shared attitudes to work or the employees' satisfaction with their jobs. It is thus an industrial design problem rather than a health problem. If sickness absence is a reflection of ill health, however, then it is a health problem with profound economic impact. It is of great importance, therefore, to determine if sickness absence is best viewed as a measure of morbidity or as a manifestation of job dissatisfaction or other problems. If it is a measure of morbidity, prevention must be preceded by knowledge of predictors.

The opportunity to study these questions comes from the Whitehall II study - a cohort of 10308 British civil servants. Men and women aged 35-55 underwent a health examination in 1985-88 and sickness absence was recorded subsequently. It may be thought that short spells of absence are less likely to reflect underlying ill health than longer spells, which require a medical certificate. In this paper, we examine the degree to which measures of health status and job satisfaction predict spells of absence of different duration, and point to major predictors of sickness absence.

\section{Methods}

STUDY POPULATION

All non-industrial civil servants aged 35-55 working in the London offices of 20 departments were invited to participate in this study. The overall response rate was $73 \%(74 \%$ for men and $71 \%$ for women). The true response rates are likely to be higher, however, because around $4 \%$ of those on the list of employees had moved before the study, and were thus not eligible for inclusion. The response rate varied by employment grade. It was $81 \%$ in the top three employment grade categories (defined below) and $68 \%$ among the lower three categories. In total, 10308 civil servants participated, of whom $66.9 \%$ (6895) were men and $33 \cdot 1 \%$ (3413) women.

BASELINE SURVEY

Between September 1985 and March 1988, participants completed questionnaires and attended a screening examination. The questionnaires provided the baseline information for this analysis. The following health status measures were recorded: the London School 
of Hygiene cardiovascular questionnaire on angina pectoris and possible myocardial infarction, ${ }^{6}$ the Medical Research Council chronic bronchitis questionnaire, ${ }^{6}$ the general household survey long-standing illness question, ${ }^{7}$ questions on past medical history of doctor-diagnosed illness, current medications, health problems over the past 12 months, symptoms over the past 14 days, self rated health status over the past 12 months, minor psychiatric morbidity, ${ }^{8}$ diagnosis of diabetes, and, in women, presence of premenstrual and menopausal symptoms. Detailed information on work characteristics was also obtained using a 67 item questionnaire. Data relating to job satisfaction have been used in this analysis. Further details of the work characteristics and other data collected have been described elsewhere. ${ }^{910}$

Information on grade of employment was obtained by asking participants to give their civil service grade at the time of the baseline survey. Changes in grade during the follow up period were not analysed in this paper. On the basis of salary, the civil service identifies 12 nonindustrial grades which, in order of decreasing salary, consist of seven "unified grades" - senior executive officers (SEOs), higher executive officers (HEOs), executive officers (EOs), clerical officers, and clerical assistants and office support staff. The term "unified grade" is used by the civil service to refer to the combination of administrative grades (previously known as permanent secretary, deputy secretary, under secretary, assistant secretary, senior principal and principal) and professional or technical staff with equivalent salaries. Similarly, the remaining professional or technical staff are combined with administrative grades (SEOs, HEOs and EOs) on the basis of salary. For analysis, to obtain significant numbers, we combined unified grades 1-6 into one category and clerical officers, clerical assistants, and office support staff into another category, thus producing six grade categories. There was a steep increment in salaries between grade categories from an annual salary in 1987 of $£ 3060-£ 6790$ in category 6 to $£ 18020-£ 62100$ in category 1. However, most of the civil servants in the top category were at the lower end of the pay scale, with $82 \%$ of men and $83 \%$ of women in category 1 earning between $£ 18020-£ 27065$. There were also marked differences in other socioeconomic indicators (education, housing tenure, car ownership, and father's occupation) in relation to grade of employment and these have been described elsewhere. ${ }^{9}$

\section{SICKNESS ABSENCE RECORDS}

Computerised sickness absence records were obtained annually from civil service pay centres. These records included the first and last dates of all absences and the reason for absence. For absences of seven days or less, civil servants were able to complete their own certificate and explain the absence. For absences longer than seven days, a medical certificate was required. Sickness absence records were checked for inconsistencies. Overlapping, consecutive, or du- plicate spells of sickness absence were merged after taking account of weekends and public holidays. This affected less than $1 \%$ of all spells of sickness absence. Most participants (93\%) gave consent for follow up based on their sickness absence records. Of these a small proportion of records ( $5 \%$ ) could not be identified. Sickness absence records of 9072 participants ( $88 \%$ of the total sample) were examined over a mean period of 20 months (range $0.3-39.6$ months).

\section{STATISTICAL ANALYSIS}

To examine the relationship between the health status measures and spells of absence of different duration we have analysed separately spells of length: 1-2 days; 3-7 days; 8-21 days; and $>21$ days. The rationale for analysing spells of different duration is that if sickness absence is influenced by both ill health and psychosocial circumstances, then we would expect ill health to be a stronger predictor of longer spells, and psychosocial circumstances to be more important predictors of shorter spells.

For each individual the number of spells of sickness absence of each type was computed and the follow up period was measured in person-years. Rates of sickness absence were computed and are expressed per 100 person years. Age adjusted rates were calculated by direct standardisation using the total study population as the standard.

Adjusted rate ratios and their 95\% confidence intervals ( $95 \% \mathrm{CI})$ were calculated ror men and women separately using Poisson regression. Details of the method used have been published previously. ${ }^{10}$ In brief, it was assumed that for each participant the occurrence of each spell followed a Poisson distribution. For shorter spells (1-2 day and 3-7 day spells) there was considerable residual variation in excess of the Poisson distribution (over dispersion). This over dispersion has no effect on the rate ratio estimates. The estimates of $95 \%$ CI were, however, adjusted for this over dispersion, the effect of which was to increase the width of the $95 \%$ CI by about $50 \%$. For longer spells (8-21 day and $>21$ day spells), no over dispersion was detected.

Individuals with incomplete data were excluded from the analyses involving those variables. Consequently, the number of individuals varied when different explanatory variables were considered. The regression models were fitted using the statistical package $G L I M^{11}$ and all other analyses were performed using the statistical package, Statistical Analysis System. ${ }^{12}$

\section{Results}

The most striking feature of sickness absence in the Civil Service is its relation to grade of employment (table 1). Among both men and women, for all spells of absence, the lower the employment grade, the higher is the rate of sickness absence. The reasons for this relation to grade of employment have been examined previously. ${ }^{10}$ Because grade is also related to other measures of health, the relation of health 
Table 1 Sickness absence rates and number of spells in relation to sex and employment grade

\begin{tabular}{|c|c|c|c|c|c|c|c|c|}
\hline \multirow[b]{3}{*}{$\begin{array}{l}\text { Men }(n=6222) \\
\text { grade }\end{array}$} & \multicolumn{8}{|c|}{ Length of sickness absence spells $(d)$} \\
\hline & \multicolumn{2}{|l|}{$1-2$} & \multicolumn{2}{|l|}{$3-7$} & \multicolumn{2}{|l|}{$8-21$} & \multicolumn{2}{|l|}{$\geq 22$} \\
\hline & Rate* & $\begin{array}{l}\text { (No of } \\
\text { spells) }\end{array}$ & Rate* & $\begin{array}{l}\text { (No of } \\
\text { spells) }\end{array}$ & Rate* & $\begin{array}{l}\text { (No of } \\
\text { spells) }\end{array}$ & Rate* & $\begin{array}{l}\text { (No of } \\
\text { spells) }\end{array}$ \\
\hline $\begin{array}{l}\text { UGI -UG6 } \\
\text { UG7 } \\
\text { SEO } \\
\text { HEO } \\
\text { EO } \\
\text { Clerical/support }\end{array}$ & $\begin{array}{r}31 \cdot 9 \\
62 \cdot 9 \\
77 \cdot 5 \\
100 \cdot 5 \\
170 \cdot 4 \\
187 \cdot 2\end{array}$ & $\begin{array}{l}(473) \\
(1543) \\
(1492) \\
(2292) \\
(7257) \\
(1619)\end{array}$ & $\begin{array}{l}11 \cdot 8 \\
22 \cdot 8 \\
28 \cdot 1 \\
39 \cdot 5 \\
54 \cdot 5 \\
75 \cdot 3\end{array}$ & $\begin{array}{l}(185) \\
(558) \\
(535) \\
(880) \\
(727) \\
(647)\end{array}$ & $\begin{array}{r}2 \cdot 8 \\
4 \cdot 4 \\
6 \cdot 1 \\
8 \cdot 9 \\
14 \cdot 1 \\
18 \cdot 4\end{array}$ & $\begin{array}{l}(46) \\
(107) \\
(117) \\
(202) \\
(186) \\
(163)\end{array}$ & $\begin{array}{r}1 \cdot 9 \\
3 \cdot 1 \\
4 \cdot 2 \\
4 \cdot 0 \\
6 \cdot 6 \\
13 \cdot 8\end{array}$ & $\begin{array}{l}(32) \\
(68) \\
(68) \\
(76) \\
(72) \\
(96)\end{array}$ \\
\hline $\begin{array}{l}\text { Women }(\mathrm{n}=2850 \\
\text { grade } \\
\text { UG1 -UG6 } \\
\text { UG7 } \\
\text { SEO } \\
\text { HEO } \\
\text { EO } \\
\text { Clerical/support }\end{array}$ & $\begin{array}{r}61 \cdot 0 \\
65 \cdot 2 \\
107 \cdot 4 \\
144 \cdot 3 \\
167 \cdot 8 \\
174 \cdot 8\end{array}$ & $\begin{array}{r}(98) \\
(250) \\
(299) \\
(966) \\
(1539) \\
(3850)\end{array}$ & $\begin{array}{l}22 \cdot 4 \\
23 \cdot 1 \\
41 \cdot 0 \\
47 \cdot 0 \\
60 \cdot 8 \\
78 \cdot 8\end{array}$ & $\begin{array}{r}(36) \\
(82) \\
(110) \\
(316) \\
(575) \\
(1800)\end{array}$ & $\begin{array}{r}7 \cdot 0 \\
3 \cdot 7 \\
9 \cdot 1 \\
17 \cdot 5 \\
19 \cdot 2 \\
27 \cdot 9\end{array}$ & $\begin{array}{l}(11) \\
(14) \\
(25) \\
(110) \\
(182) \\
(657)\end{array}$ & $\begin{array}{r}3 \cdot 0 \\
2 \cdot 9 \\
2 \cdot 1 \\
7 \cdot 6 \\
11 \cdot 3 \\
15.9\end{array}$ & $\begin{array}{r}(4) \\
(11) \\
(7) \\
(41) \\
(85) \\
(289)\end{array}$ \\
\hline
\end{tabular}

* Age adjusted rates expressed per 100 person years.

$\mathrm{UG}=$ unified grades; $\mathrm{SEO}=$ senior executive officer, $\mathrm{HEO}=$ higher executive officer; $\mathrm{EO}=$ executive officer

Table 2 Rate ratios of different lengths of sickness absence associated with a 10 year increase in age

\begin{tabular}{llllll}
\hline \multirow{2}{*}{$\begin{array}{l}\text { Length of sickness } \\
\text { absence spells }(\mathrm{d})\end{array}$} & \multicolumn{3}{l}{ Men } & & Women \\
\cline { 2 - 3 } \cline { 5 - 6 } \cline { 5 - 6 } & $\mathrm{RR}^{*}$ & $(95 \% \mathrm{CI})$ & & $\mathrm{RR}^{*}$ & $(95 \% \mathrm{CI})$ \\
\hline $1-2$ & 1.00 & $(0.95,1.05)$ & & 0.84 & $(0.79,0.90)$ \\
$3-7$ & 1.05 & $(0.99,1.11)$ & & 1.01 & $(0.94,1.09)$ \\
$8-21$ & 1.16 & $(1.04,1.30)$ & & 1.14 & $(1.03,1.27)$ \\
$\geq 22$ & 1.62 & $(1.38,1.90)$ & & 1.38 & $(1.17,1.63)$ \\
\hline
\end{tabular}

* Rate ratios (95\% confidence intervals $(\mathrm{CI})$ ) adjusted for grade of employment

measures to sickness absence in this paper is presented adjusted for grade.

Age was a strong predictor of morbidity. Table 2 shows the slope of the relationship between sickness absence spells of different duration and age. The rate ratios show the relative change in rates for each length of spell of absence associated with a 10 year increase in age. There is no association in men, and a negative association in women, between spells of sickness absence of 1-2 days' duration and age. However, the association becomes positive and stronger with increasing length of spell.

The relationship between baseline measures of health and rates of spells of absence of different duration among men and women are shown in tables 3 and 4 . In general, the longer the duration of absence, the more strongly did the baseline measures of health predict rates of absence. However, most of the health measures that predicted longer spells of absence also predicted shorter spells, albeit to a lesser extent. The association between self reported health and sickness absence was particularly no-

Table 3 Rate ratios of different lengths of sickness absence in relation to measures of health status in men

\begin{tabular}{|c|c|c|c|c|c|c|c|c|c|}
\hline \multirow[b]{3}{*}{ Measures } & & \multicolumn{8}{|c|}{ Length of sickness spells (d) } \\
\hline & & \multicolumn{2}{|l|}{$1-2$} & \multicolumn{2}{|l|}{$3-7$} & \multicolumn{2}{|l|}{$8-21$} & \multicolumn{2}{|l|}{$\geq 22$} \\
\hline & & $\begin{array}{l}R R^{*} \\
(95 \% C I)\end{array}$ & $\begin{array}{l}\text { No of } \\
\text { spells }\end{array}$ & $\begin{array}{l}R R^{*} \\
(95 \% C I)\end{array}$ & $\begin{array}{l}\text { No of } \\
\text { spells }\end{array}$ & $\begin{array}{l}R R^{*} \\
(95 \% C I)\end{array}$ & $\begin{array}{l}\text { No of } \\
\text { spells }\end{array}$ & $\begin{array}{l}R R^{*} \\
(95 \% C I)\end{array}$ & $\begin{array}{l}\text { No of } \\
\text { spells }\end{array}$ \\
\hline $\begin{array}{l}\text { Probable/possible ischaemia } \\
\text { on ECG }\end{array}$ & $\begin{array}{l}\text { No } \\
\text { Yes }\end{array}$ & $\begin{array}{l}1 \cdot 0 \\
1.03 \\
(0.92,1 \cdot 16)\end{array}$ & $\begin{array}{r}(9001) \\
(670)\end{array}$ & $\begin{array}{l}1 \cdot 0 \\
1 \cdot 11 \\
(0 \cdot 97,1 \cdot 27)\end{array}$ & $\begin{array}{r}(3263) \\
(266)\end{array}$ & $\begin{array}{l}1 \cdot 0 \\
1.06 \\
(0 \cdot 82,1 \cdot 38)\end{array}$ & $\begin{array}{r}(758) \\
(62)\end{array}$ & $\begin{array}{l}1 \cdot 0 \\
1 \cdot 89 \\
(1 \cdot 42,2 \cdot 53)\end{array}$ & $\begin{array}{r}(357) \\
(55)\end{array}$ \\
\hline Angina by questionnaire & $\begin{array}{l}\text { No } \\
\text { Yes }\end{array}$ & $\begin{array}{l}1 \cdot 0 \\
1 \cdot 37 \\
(0 \cdot 16,1 \cdot 61)\end{array}$ & $\begin{array}{r}(9199) \\
(331)\end{array}$ & $\begin{array}{l}1 \cdot 0 \\
1 \cdot 47 \\
(1 \cdot 22,1 \cdot 77)\end{array}$ & $\begin{array}{r}(3345) \\
(131)\end{array}$ & $\begin{array}{l}1 \cdot 0 \\
1 \cdot 26 \\
(0 \cdot 86,1 \cdot 85)\end{array}$ & $\begin{array}{r}(777) \\
(27)\end{array}$ & $\begin{array}{l}1 \cdot 0 \\
1 \cdot 61 \\
(1 \cdot 00,2 \cdot 59)\end{array}$ & $\begin{array}{r}(387) \\
(18)\end{array}$ \\
\hline History of diabetes & $\begin{array}{l}\text { No } \\
\text { Yes }\end{array}$ & $\begin{array}{l}1 \cdot 0 \\
1 \cdot 19 \\
(0 \cdot 92,1 \cdot 54)\end{array}$ & $\begin{array}{r}(9433) \\
(130)\end{array}$ & $\begin{array}{l}1 \cdot 0 \\
1 \cdot 22 \\
(0 \cdot 90,1 \cdot 65)\end{array}$ & $\begin{array}{r}(3458) \\
(48)\end{array}$ & $\begin{array}{l}1 \cdot 0 \\
1.54 \\
(0.92,2 \cdot 57)\end{array}$ & $\begin{array}{r}(796) \\
(15)\end{array}$ & $\begin{array}{l}1 \cdot 0 \\
0.98 \\
(0 \cdot 41,2 \cdot 38)\end{array}$ & $\begin{array}{r}(405) \\
(5)\end{array}$ \\
\hline Self reported health & $\begin{array}{l}\text { Very good } \\
\text { Good } \\
\text { Average } \\
\text { Poor }\end{array}$ & $\begin{array}{l}1 \cdot 0 \\
1 \cdot 31 \\
(1 \cdot 21,1 \cdot 41) \\
1 \cdot 70 \\
(1 \cdot 56,1 \cdot 84) \\
2 \cdot 30 \\
(2 \cdot 04,2 \cdot 60)\end{array}$ & $\begin{array}{r}(2419) \\
(3946) \\
(2527) \\
(748)\end{array}$ & $\begin{array}{l}1 \cdot 0 \\
1 \cdot 38 \\
(1 \cdot 26,1.51) \\
1 \cdot 76 \\
(1 \cdot 59,1.94) \\
2 \cdot 53 \\
(2 \cdot 19,2.92)\end{array}$ & $\begin{array}{r}(852) \\
(1463) \\
(917) \\
(290)\end{array}$ & $\begin{array}{l}1 \cdot 0 \\
1 \cdot 38 \\
(1 \cdot 15,1 \cdot 66) \\
2 \cdot 11 \\
(1 \cdot 74,2 \cdot 57) \\
4 \cdot 12 \\
(3 \cdot 21,5 \cdot 27)\end{array}$ & $\begin{array}{l}(176) \\
(307) \\
(235) \\
(100)\end{array}$ & $\begin{array}{l}1 \cdot 0 \\
2 \cdot 25 \\
(1 \cdot 69,2 \cdot 99) \\
2 \cdot 78 \\
(2 \cdot 04,3 \cdot 79) \\
6 \cdot 15 \\
(4 \cdot 27,8 \cdot 88)\end{array}$ & $\begin{array}{r}(64) \\
(182) \\
(110) \\
(54)\end{array}$ \\
\hline $\begin{array}{l}\text { Regular cough with } \\
\text { phlegm in winter }\end{array}$ & $\begin{array}{l}\text { No } \\
\text { Yes }\end{array}$ & $\begin{array}{l}1 \cdot 0 \\
1 \cdot 24 \\
(1 \cdot 13,1 \cdot 36)\end{array}$ & $\begin{array}{l}(8477) \\
(1077)\end{array}$ & $\begin{array}{l}1 \cdot 0 \\
1 \cdot 21 \\
(1 \cdot 08,1 \cdot 36)\end{array}$ & $\begin{array}{r}(3114) \\
(389)\end{array}$ & $\begin{array}{l}1.0 \\
1.57 \\
(1 \cdot 29,1.91)\end{array}$ & $\begin{array}{l}(700) \\
(115)\end{array}$ & $\begin{array}{l}1 \cdot 0 \\
1 \cdot 57 \\
(1 \cdot 02,1 \cdot 84)\end{array}$ & $\begin{array}{r}(358) \\
(51)\end{array}$ \\
\hline Long standing illness & $\begin{array}{l}\text { No } \\
\text { Yes }\end{array}$ & $\begin{array}{l}1 \cdot 0 \\
1 \cdot 29 \\
(1 \cdot 20,1 \cdot 39)\end{array}$ & $\begin{array}{l}(4339) \\
(2558)\end{array}$ & $\begin{array}{l}1 \cdot 0 \\
1 \cdot 25 \\
(1 \cdot 14,1 \cdot 36)\end{array}$ & $\begin{array}{r}(1557) \\
(893)\end{array}$ & $\begin{array}{l}1.0 \\
1.67 \\
(1.41,1.98)\end{array}$ & $\begin{array}{l}(305) \\
(239)\end{array}$ & $\begin{array}{l}1 \cdot 0 \\
2 \cdot 19 \\
(1 \cdot 73,2 \cdot 79)\end{array}$ & $\begin{array}{l}(131) \\
(142)\end{array}$ \\
\hline $\begin{array}{l}\text { No of health problems } \\
\text { in the last year }\end{array}$ & $\begin{array}{l}\text { None } \\
1\end{array}$ & $\begin{array}{l}1 \cdot 0 \\
1 \cdot 24 \\
(1 \cdot 12,1 \cdot 36)\end{array}$ & $\begin{array}{l}(1760) \\
(2007)\end{array}$ & $\begin{array}{l}1 \cdot 0 \\
1 \cdot 27 \\
(1 \cdot 13,1 \cdot 43)\end{array}$ & $\begin{array}{l}(588) \\
(692)\end{array}$ & $\begin{array}{l}1 \cdot 0 \\
1 \cdot 28 \\
(1.01,1.63)\end{array}$ & $\begin{array}{l}(121) \\
(143)\end{array}$ & $\begin{array}{l}1 \cdot 0 \\
1 \cdot 89 \\
(1 \cdot 36,2 \cdot 63)\end{array}$ & $\begin{array}{l}(55) \\
(96)\end{array}$ \\
\hline & $\begin{array}{l}2-4 \\
>4\end{array}$ & $\begin{array}{l}1.48 \\
(1 \cdot 36,1 \cdot 62) \\
2 \cdot 24 \\
(1 \cdot 87,2 \cdot 68)\end{array}$ & $\begin{array}{r}(2801) \\
(307)\end{array}$ & $\begin{array}{l}1.63 \\
(1.46,1.81) \\
2 \cdot 94 \\
(2 \cdot 40,3.59)\end{array}$ & $\begin{array}{r}(1037) \\
(135)\end{array}$ & $\begin{array}{l}1 \cdot 81 \\
(1 \cdot 46,2 \cdot 25) \\
3 \cdot 71 \\
(2 \cdot 56,5 \cdot 37)\end{array}$ & $\begin{array}{r}(239) \\
(37)\end{array}$ & $\begin{array}{l}1 \cdot 63 \\
(1 \cdot 17,2 \cdot 26) \\
3 \cdot 65 \\
(2 \cdot 15,6 \cdot 18)\end{array}$ & $\begin{array}{r}(101) \\
(19)\end{array}$ \\
\hline Psychiatric symptoms & $\begin{array}{l}\text { No } \\
\text { Yes }\end{array}$ & $\begin{array}{l}1 \cdot 0 \\
1 \cdot 27 \\
(1 \cdot 19,1 \cdot 45)\end{array}$ & $\begin{array}{l}(6765) \\
(2860)\end{array}$ & $\begin{array}{l}1 \cdot 0 \\
1 \cdot 34 \\
(1 \cdot 24,1 \cdot 45)\end{array}$ & $\begin{array}{l}(2429) \\
(1083)\end{array}$ & $\begin{array}{l}1 \cdot 0 \\
1 \cdot 47 \\
(1 \cdot 27,1 \cdot 70)\end{array}$ & $\begin{array}{l}(550) \\
(265)\end{array}$ & $\begin{array}{l}1 \cdot 0 \\
1 \cdot 46 \\
(1 \cdot 19,1 \cdot 80)\end{array}$ & $\begin{array}{l}(281) \\
(131)\end{array}$ \\
\hline $\begin{array}{l}\text { Drug therapy for } \\
\text { hypertension }\end{array}$ & $\begin{array}{l}\text { No } \\
\text { Yes }\end{array}$ & $\begin{array}{l}1 \cdot 0 \\
1 \cdot 40 \\
(1 \cdot 29,1 \cdot 52)\end{array}$ & $\begin{array}{r}(8997) \\
(339)\end{array}$ & $\begin{array}{l}1 \cdot 0 \\
1 \cdot 10 \\
(0 \cdot 91,1 \cdot 34)\end{array}$ & $\begin{array}{r}(3289) \\
(128)\end{array}$ & $\begin{array}{l}1 \cdot 0 \\
1 \cdot 25 \\
(0 \cdot 89,1 \cdot 75)\end{array}$ & $\begin{array}{r}(765) \\
(37)\end{array}$ & $\begin{array}{l}1 \cdot 0 \\
1 \cdot 78 \\
(1 \cdot 21,2 \cdot 62)\end{array}$ & $\begin{array}{r}(376) \\
(29)\end{array}$ \\
\hline Current smoker & $\begin{array}{l}\text { No } \\
\text { Yes }\end{array}$ & $\begin{array}{l}1 \cdot 0 \\
1 \cdot 09 \\
(1 \cdot 01,1 \cdot 18)\end{array}$ & $\begin{array}{l}(7682) \\
(1985)\end{array}$ & $\begin{array}{l}1 \cdot 0 \\
1 \cdot 29 \\
(1 \cdot 19,1 \cdot 41)\end{array}$ & $\begin{array}{r}(2704) \\
(823)\end{array}$ & $\begin{array}{l}1 \cdot 0 \\
1.42 \\
(1 \cdot 21,1.67)\end{array}$ & $\begin{array}{l}(611) \\
(210)\end{array}$ & $\begin{array}{l}1 \cdot 0 \\
1 \cdot 29 \\
(1 \cdot 01,1 \cdot 63)\end{array}$ & $\begin{array}{r}(317) \\
(95)\end{array}$ \\
\hline
\end{tabular}


Table 4 Rate ratios of different lengths of sickness absence in relation to measures of health status in women

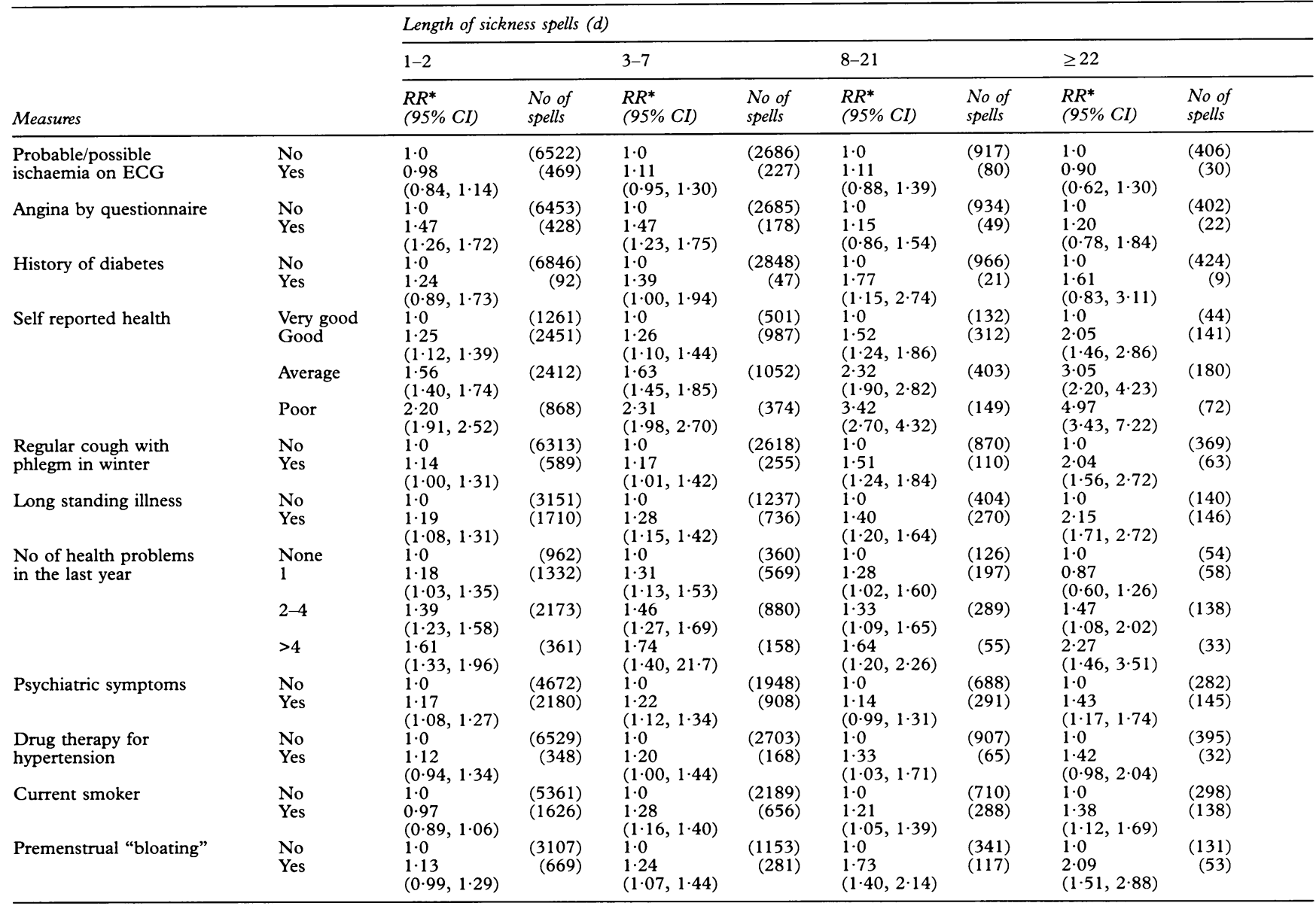

${ }^{*}$ Rate ratios $(95 \%$ confidence intervals $(\mathrm{CI})$ ) adjusted for age and grade of employment.

Table 5 Rate ratios of different lengths of sickness absence in relation to self rated health status in men

\begin{tabular}{|c|c|c|c|c|c|}
\hline \multirow[b]{3}{*}{ Adjustments } & \multicolumn{5}{|c|}{ Length of sickness absence spells $(d)$} \\
\hline & \multirow{2}{*}{$\begin{array}{l}\text { Self rated } \\
\text { health }\end{array}$} & \multicolumn{2}{|l|}{$1-2$} & \multicolumn{2}{|l|}{$3-7$} \\
\hline & & $R R$ & $(95 \% C I)$ & $R R$ & $(95 \% C I)$ \\
\hline Age, grade & $\begin{array}{l}\text { Very good } \\
\text { Good } \\
\text { Average } \\
\text { Poor }\end{array}$ & $\begin{array}{l}1 \cdot 0 \\
1 \cdot 30 \\
1 \cdot 69 \\
2 \cdot 31\end{array}$ & $\begin{array}{l}(1.21,1.40) \\
(1.56,1.84) \\
(2.05,2.61)\end{array}$ & $\begin{array}{l}1.0 \\
1.37 \\
1.73 \\
2.52\end{array}$ & $\begin{array}{l}(1.25,1.50) \\
(1.57,1.92) \\
(2.19,2.91)\end{array}$ \\
\hline $\begin{array}{l}\text { Age, grade, rate of long } \\
\text { spells of absence }\end{array}$ & $\begin{array}{l}\text { Very good } \\
\text { Good } \\
\text { Average } \\
\text { Poor }\end{array}$ & $\begin{array}{l}1 \cdot 0 \\
1 \cdot 28 \\
1.63 \\
2 \cdot 15\end{array}$ & $\begin{array}{l}(1 \cdot 19,1 \cdot 38) \\
(1.50,1 \cdot 77) \\
(1 \cdot 89,2 \cdot 43)\end{array}$ & $\begin{array}{l}1 \cdot 0 \\
1.32 \\
1.60 \\
2 \cdot 08\end{array}$ & $\begin{array}{l}(1.21,1.44) \\
(1.45,1.77) \\
(1.79,2.41)\end{array}$ \\
\hline
\end{tabular}

Subjects with sickness absence records of less than six months have been excluded.

ticeable, especially for longer spells of absence.

The relationship between baseline measures of health and short spells of absence may partly be explained by the same individuals having higher rates of absence for both short and long spells. Table 5 shows the association between self rated health and short spells of 1-2 days and 3-7 days in men before and after adjusting for the rate of long spells of sickness absence. The figures show that after adjusting for the rate of long spells of absence, the association between self rated health and short spells was only slightly reduced. Similar results were seen in women (not shown).

Table 6 shows the relationship between job satisfaction and rates of spells of absence of different duration. Job satisfaction was more strongly associated with spells of 1-2 days than with longer spells of absence.

We have attempted to remove that part of the association that may be due to health status by adjusting for self reported health. Table 6 shows that in both men and women, the association between job satisfaction and longer spells of absence was greatly attenuated, resulting in a minimal association between job satisfaction and spells of absence of 3 days or more in length. In contrast, the strong association between job satisfaction and spells of 1-2 days remained after adjusting for self reported health.

Adjustment for job satisfaction had little effect on the relationship between self reported health and spells of sickness absence as reported in tables 3 and 4 . The rate ratios for short spells of absence decreased by less than $5 \%$ and, in women, the rate ratios for long spells of absence increased, but again by less than $5 \%$.

\section{Discussion}

The call from WHO to consider health in terms of social, physical, and mental wellbeing ${ }^{13}$ has inspired considerable comment, much of it critical, and only some attempt to operationalise it. ${ }^{14}$ Interestingly, some move towards this has come from the "outcomes" movement - the desire to evaluate the outcome of medical care not only in terms of disease 
Table 6 Rate ratios of different lengths of sickness absence in relation to job satisfaction in men and women

\begin{tabular}{|c|c|c|c|c|c|}
\hline & \multicolumn{5}{|c|}{ Length of sickness absence spells (d) } \\
\hline & $\begin{array}{l}\text { Fob } \\
\text { satisfaction* }\end{array}$ & $\begin{array}{l}1-2 \\
R R \dagger \\
(95 \% C I)\end{array}$ & $\begin{array}{l}3-7 \\
R R \dagger \\
(95 \% C I)\end{array}$ & $\begin{array}{l}8-21 \\
R R \dagger \\
(95 \% C I)\end{array}$ & $\begin{array}{l}\geq 22 \\
R R \dagger \\
(95 \% C I)\end{array}$ \\
\hline \multicolumn{6}{|l|}{$\begin{array}{l}\text { Men } \\
\text { Adjustments: }\end{array}$} \\
\hline & $\begin{array}{l}\text { High } \\
\text { Low }\end{array}$ & $\begin{array}{l}1 \cdot 0 \\
1 \cdot 33 \\
(1 \cdot 25,1 \cdot 42)\end{array}$ & $\begin{array}{l}1 \cdot 0 \\
1 \cdot 15 \\
(1 \cdot 04,1 \cdot 27)\end{array}$ & $\begin{array}{l}1 \cdot 0 \\
1.06 \\
(0.92,1.22)\end{array}$ & $\begin{array}{l}1 \cdot 0 \\
1 \cdot 28 \\
(1 \cdot 05,1 \cdot 56)\end{array}$ \\
\hline $\begin{array}{l}\text { Age, grade, self } \\
\text { reported health }\end{array}$ & $\begin{array}{l}\text { High } \\
\text { Low }\end{array}$ & $\begin{array}{l}1 \cdot 0 \\
1 \cdot 26 \\
(1 \cdot 18,1 \cdot 34)\end{array}$ & $\begin{array}{l}1 \cdot 0 \\
1 \cdot 08 \\
(0.97,1 \cdot 20)\end{array}$ & $\begin{array}{l}1 \cdot 0 \\
0 \cdot 96 \\
(0 \cdot 84,1 \cdot 11)\end{array}$ & $\begin{array}{l}1 \cdot 0 \\
1 \cdot 14 \\
(0 \cdot 94,1 \cdot 40)\end{array}$ \\
\hline $\begin{array}{l}\text { Women } \\
\text { Adjustments: }\end{array}$ & & & & & \\
\hline Age, grade & $\begin{array}{l}\text { High } \\
\text { Low }\end{array}$ & $\begin{array}{l}1 \cdot 0 \\
1 \cdot 27 \\
(1 \cdot 18,1 \cdot 37)\end{array}$ & $\begin{array}{l}1 \cdot 0 \\
1 \cdot 13 \\
(1 \cdot 00,1 \cdot 27)\end{array}$ & $\begin{array}{l}1 \cdot 0 \\
1 \cdot 16 \\
(1 \cdot 02,1 \cdot 31)\end{array}$ & $\begin{array}{l}1 \cdot 0 \\
0 \cdot 98 \\
(0 \cdot 80,1 \cdot 19)\end{array}$ \\
\hline $\begin{array}{l}\text { Age, grade, self } \\
\text { reported health }\end{array}$ & $\begin{array}{l}\text { High } \\
\text { Low }\end{array}$ & $\begin{array}{l}1 \cdot 0 \\
1 \cdot 21 \\
(1 \cdot 12,1 \cdot 30)\end{array}$ & $\begin{array}{l}1 \cdot 0 \\
1 \cdot 06 \\
(0 \cdot 94,1 \cdot 19)\end{array}$ & $\begin{array}{l}1 \cdot 0 \\
1.05 \\
(0 \cdot 92,1 \cdot 19)\end{array}$ & $\begin{array}{l}1 \cdot 0 \\
0 \cdot 87 \\
(0 \cdot 71,1 \cdot 06)\end{array}$ \\
\hline
\end{tabular}

* Job satisfaction levels classified as above and below the median score.

† Rate ratios $(95 \%$ confidence intervals $(\mathrm{CI})$ ).

abatement but in terms of social and physical functioning and perceived wellbeing. ${ }^{15}$

It is in this context that sickness absence should be seen. It may be a socially biased and prescriptive view, but if healthy functioning for people in stable jobs is, by definition, attending for work; then absence from work indicates some lack of functioning - whether the causes are psychological, social or physical.

Sickness absence rates cannot be used uncritically as health measures. For example, countries as similar in health and socioeconomic level as Belgium and The Netherlands have differences in sickness absence rates that are likely to be due to differences in social security and other policies. ${ }^{16}$ Similarly, time trends in absence rates in Sweden may relate to policy changes. ${ }^{17}$ The question this paper addresses is the extent to which health is a predictor of sickness absence rates within one jurisdiction.

In the Whitehall II study, we have shown that three sets of factors predict sickness absence rates: personal factors such as age, sex, and health behaviours; social circumstances outside work; and aspects of the way work is organised. ${ }^{10}$ Other studies have identified a number of risk factors: age, sex, socioecomonic status, type of employment, length of employment, and social circumstances. ${ }^{18-20} \mathrm{Al}-$ though sickness absence is, by definition, absence from work attributable to sickness, very few studies have examined the extent to which health predicts rates of sickness absence, and more specifically rates of absence of different duration. ${ }^{1820}$

If sickness absence is a measure of ill health, then rates might be expected to be higher in individuals with other indicators or predictors of ill health. ${ }^{182021}$ In the data presented, age was not related to rates of spells of absence of 1-2 days, but was more strongly and significantly related to rates of longer spells of absence. Similarly, most measures of health were more strongly associated with rates of longer spells of absence than shorter spells. While longer spells of absence may be better indicators of ill health, short spells are not determined by social or psychological factors alone. Most of the health measures that pre- dicted longer spells also predicted shorter spells, albeit to a lesser extent. For some, such as angina symptoms, self rated health and reported health problems in the last year, there was a strong relationship with both long and short spells of sickness absence. This association was virtually unchanged when adjustment was made for the possibility that some individuals may be prone to both short and long spells of absence.

To some extent, sickness absence may be a reflection of employees' perceptions of their health and their behaviour in response to illness rather than physical disease. In other words, those who have a tendency to complain may also have a greater tendency to take time off work than others who deny the presence of illness. A number of studies have reported associations between perceived ill health and sickness absence. ${ }^{102-24}$ However, Semmence, ${ }^{25}$ in several studies in the 1960 s and 1970 s, found that sickness absence was a reliable indicator of subsequent serious morbidity and medical retirements.

While illness behaviour and reporting bias probably contribute to sickness absence, they are unlikely to explain the strong associations which increase for spells of longer duration. Firstly, in other studies, self reported health is a strong predictor of mortality, ${ }^{26}$ which suggests it reflects "real" pathology. Secondly, the questions on angina pectoris and winter cough and phlegm were quite specific and less likely to be influenced by a general tendency to complain.

A relationship between minor psychiatric morbidity as measured by the general health questionnaire $^{8}$ and sickness absence was reported by Jenkins ${ }^{27}$ in a study of executive officers in the civil service, especially for spells of absence of more than seven days. A significant increase in illness and disease was also found in a Swedish study which followed participants over a 10 year period: those with a high psychiatric score were significantly more likely to develop cancer and other diseases. ${ }^{28}$ A current debate is whether people who have minor psychiatric illness are more likely to report health problems, or whether having a psychiatric illness predisposes people to develop ill health? It has been argued that there 
is a strong positive association between physical and psychiatric disorders. ${ }^{29}$ The health and lifestyle survey found that reports of minor physical illness were significantly related to affective state in both men and women. ${ }^{30}$ Whatever the causal direction, minor psychiatric morbidity is clearly related to measures of ill health and to sickness absence.

Might the findings from this sample of civil servants be in some way atypical? The sample was selected in several ways. It excluded people permanently absent from work due to unemployment or illness; it was confined to office workers in public service; and the overall response rate was $73 \%$, which excluded about one-quarter due to non-response. Non-responders have been shown in other studies to be less healthy. ${ }^{31}$ These factors should have reduced the level of ill health and disease in the study population compared with the general population. The most likely effect of these sources of selection bias, would have been to reduce the chance of observing the reported differences, but it is unlikely that this would change the relationship, within the cohort followed, between baseline levels of health and subsequent sickness absence rates.

Sickness absence is often considered to be a useful indicator of the quality of the work and home environment. Job satisfaction is likely to reflect a number of aspects of the work and home environment. Working conditions may influence the incidence and prevalence of disease $^{32}$ and thereby affect sickness absence rates. Alternatively, there may be a more "direct" effect on sickness absence independent of effects on health status. This might be the explanation for the data in tables 5 and 6 . The association between job satisfaction and short spells of absence is not affected by adjusting for health status; the association with long spells is reduced appreciably. This suggests that the effect of working conditions on long spells of absence may be related to the effect of work on health. This will be explored more fully in a separate report.

It is also likely to relate more strongly to short spells of absence, rather than longer spells, and more likely to be independent of health status. The data presented provide some support for this assertion, when adjustment for self-rated health status had a minimal effect on the strong relationship between low job satisfaction and rates of spells of absence for 1-2 days.

The estimate of the size of association between measures of health and sickness absence may be conservative. All the analyses were adjusted for grade of employment, because of the strength of the association between grade and sickness absence rates. ${ }^{10}$ If part of the reason for the higher rates of absence in lower grades is that they do have more ill health, then adjusting for grade is likely to underestimate the association between measures of ill health and sickness absence rates.

In addition to examining spells of different duration, it would be useful to examine spells with different reasons for absence. We are currently collecting data on diagnosis of certified absence and will be able to investigate further the relationship between baseline health and different disease categories.

We conclude that long spells of absence, greater than seven days, are likely to be health related. This is consistent with earlier studies. ${ }^{182025}$ From our data, it seems that ill health also plays an important part in shorter spells of absence. Nevertheless, studies concerned with ill health resulting in a medical diagnosis do well to focus on longer spells of absence. It may be, however, that the attempt to disaggregate a physical health component from the psychological and social elements is artificial. Grade of employment, for example is almost equally related to long spells, which are better predicted by health, and to short spells that are less well predicted. If healthy functioning is a mixture of social, psychological and physical functioning, then sickness absence may serve well as an integrated measure of functioning. It may therefore be useful as an "end point" in studies of the determinants of healthy functioning in working populations.

We thank all participating civil service departments and their We thank all participating civil service departments and their Occupational Health Service, Dr George Sorrie and Dr Adrian Occupational Health Service, Dr George Sorrie and Dr Adrian Demmence; the Civil Service Central Monitoring Service and Dr Frank O'Hara; the Council of Civil Service Unions; and all participating civil servants. We would like to thank all members
of the Whitehall II study team, and in particular Jenny Head of the Whitehall II study team, and in particular Jenny Head
and Alan Harding for computer support and screening coordinator Julie Moore.

The study was supported by the Medical Research Council Health and Safety Executive, British Heart Foundation, Civil Service Occupational Health Service, National Heart Lung and Blood Institute (2 RO1 HL36310-04), Agency for Health Care Policy Research (5 RO1 HS06516), The New England Medical Centre - Division of Health Improvement, Ontario Workers' Centre - Division of Health Improvement, Ontario Workers Compensation Institute, and The John D and Catherine T MacArthur Foundation Research Network on Successful Midlife Development. Fiona North was supported by the Medical Research Council of New
ation of New Zealand.

1 Central Statistical Office. Regional trends. Vol 20. London: HM Stationery Office, 1985 .

2 Confederation of British Industry. Too much time out? $C B I$ Percom survey on absence from work. London: CBI, 1993.

3 Anonymous. Sickness absence in hospital staff. (Editorial). Lancet 1979;ii:1278-1279.

4 Johns G, Nicholson N. The meaning of absence: new strategies for theory and research. In: Research in Organizational Behaviour 1982;4:127-72.

5 Nicholson N, Johns G. The absence culture and the psychological contract - who's in control of absence? Academy of Management Review 1985;10:397-407.

6 Rose GA, Blackburn H, Gillum RF, Prineas RJ. Cardiovascular survey methods. Geneva: WHO, 1982.

7 Office Population Censuses and Surveys. General household survey 1977. London: HMSO, 1979.

8 Goldberg DP. In: The detection of psychiatric illness by questionnaire. London: Oxford University Press, 1972.

9 Marmot MG, Davey Smith G, Stansfield S, et al. Health inequalities among British civil servants: the Whitehall II study. Lancet 1991;337:1387-93.

10 North F, Syme SL, Feeney A, Head J, Shipley MJ, Marmot MG. Explaining socioeconomic differences in sicknes absence: the Whitehall II study. BMF 1993;306:361-6.

11 Numerical Algorithms Group. The GLIM system release 3.77 Manual. Oxford: 1987.

12 SAS Institute. SAS user's guide. Cary NC, SAS Institute Inc, 1985.

13 World Health Organisation. Health for all by the year 2000 Copenhagen: World Health Organisation, Regional Office for Europe, 1986.

14 Berkman LF, Breslow L. Health and ways of living - The Alameda county study. New York, Oxford: Oxford University Press, 1983.

15 Ware JE. Measuring patient function and well-being: some lessons from the medical outcomes study. In: Heithoff $\mathrm{KA}$, Lohr KN, eds. Effectiveness and outcomes in health care. Washington D.C. National Academy Press, 1990; 107-119.

16 Prins R, Graaf De A. Comparison of sickness absence in Belgium, German, and Dutch Firms. Br F Ind Med 1986; 43:529-36.

17 Alexanderson K, Leijon M, Akerlind I, Rydh H, Bjurulf P. Epidemiology of sickness absence in a Swedish county in 1985, 1986 and 1987. Scand $\mathcal{F}$ Soc Med 1994;22:27-34. 
18 Taylor PH. Occupational and regional associations of death, disablement and sickness absence among Post Office staff 1972-75. Br f Ind Med 1976;33:230-35.

19 McKeown KD. Sickness absence. $尹 R$ Soc Med 1989;82: 188-9.

20 Chevalier A, Luce D, Blanc C, Goldberg M. Sickness absence at the French National Electric and Gas Company. Br F Ind Med 1987;44:101-10.

21 Searle SJ. Sickness absence and duration of service in the Post Office 1982-3. Br f Ind Med 1986;43:458-64.

22 Taylor PJ. Personal factors associated with sickness absence. Br f Ind Med 1968;25:106-17.

23 McKenna SP, Hunt SM, McEwen J. Absence from work and perceived health among mine rescue workers. $f$ Occup Med 1981;31:151-7.

24 Kristensen TS. Sickness absence and work strain among Danish slaughterhouse workers: an analysis of absence from work regarded as coping behaviour. Soc Sci Med 1991;32:15-27.
25 Semmence AM. The politics of occupational medicine. $\mathcal{f} R$ Soc Med 1994;80:668-73.

26 Idler EL, Angel RJ. Self-rated health and mortality in the NHANES-I epidemiologic follow-up study. Am $₹$ Public Health 1990;80:446-52.

27 Jenkins R. Minor psychiatric morbidity in employed young men and women and its contribution to sickness absence. Br 7 Ind Med 1984;42:147-54.

28 Hagnell O. Ann N Y Acad Sci 1966;125:846.

29 Eastwood MR, Trevelyan MH. Relationship between physical and psychiatric disorder. Psychol Med 1972;2:363-72

30 Cox BD, Blaxter M, Buckle AJL, et al. The health and Cox BD, Blaxter M, Buckle AJL, et al. The health and
lifestyle survey: Preliminary report. Cambridge: Health lifestyle survey: Preliminary report.

31 Alderson M. An introduction to epidemiology. Macmillan: London and Basingstoke, 1976.

32 Karasek R. Theorell T. Healthy work: Stress, productivity and the reconstruction of working life. New York: Basic Books, 1990. 